\title{
An enhanced hybrid genetic algorithm for solving traveling salesman problem
}

\author{
Zeravan Arif Ali, Subhi Ahmed Rasheed, Nabeel No'man Ali \\ ${ }^{1}$ Management Information System, Department at Duhok Polytechnic University, Iraq
}

\begin{abstract}
Article Info
ABSTRACT

Article history:

Received Aug 7, 2019

Revised Nov 9, 2019

Accepted Nov 23, 2019

Keywords:

Genetic algorithm

Local search

TSP

Robust known the exceedingly famed NP-hard problem in combinatorial optimization is the Traveling Salesman Problem (TSP), promoting the skillful algorithms to get the solution of TSP have been the burden for several scholars. For inquiring global optimal solution, the presented algorithm hybridizes genetic and local search algorithm to take out the uplifted quality results. The genetic algorithm gives the best individual of population by enhancing both cross over and mutation operators while local search gives the best local solutions by testing all neighbor solution. By comparing with the conventional genetic algorithm, the numerical outcomes acts that the presented algorithm is more adequate to attain optimal or very near to it. Problems arrested from the TSP library strongly trial the algorithm and shows that the proposed algorithm can reap outcomes within reach optimal. For more details, please download TEMPLATE HELP FILE from the website.
\end{abstract}

Copyright (C) 2020 Institute of Advanced Engineering and Science. All rights reserved.

\section{Corresponding Author:}

Zeravan Arif Ali,

Duhok Polytecgnic University,

Department at Duhok Polytechnic University, Iraq.

Email: dqszeebaree2@live.utm.my

\section{INTRODUCTION}

The Traveling Salesman Problem [1] is the renowned and considerable combinatorial optimization problems. It was examining in theoretical computer science and operational research fields. Ever after there is no solution near and proximal to constant, consequently the traveling salesman problem is NP complete. As in definition of the TSP, the main idea says there several cities and the distance among each couple of it. The goal act to return to the root city afterword visiting every city nicely at one time. The challenge is to calculate and minimize the total value of the cost during traveling [2-3]. The test and difficulty in the traveling salesman problem something curious, so that researchers make it as a podium in order to examine both the exact and approximation algorithms. The exact algorithms are good for the small problems, while they are not for the large range problems, because the performance is decreased, and the time need to get the optimal one is long [4]. That is why the other types of algorithms are better and they provide elegant results. There are two types of optimal solutions, one of them is local while the other is global, and both solutions can get by using the approximation algorithms [5]. Algorithms used to find optimum called heuristic optimization. Different kinds of heuristic algorithms have been handled to lead to get the local optimal solution and try to gain the global optimal solution or near global solution to solve TSP, like Genetic Algorithm [5-6], Simulated Annealing [7], Particle Swarm [8], Tabu Search [9], Artificial Neural Network [9-10] Ant Colony [10], Artificial Immune algorithm, and genetic algorithm [11].

GA concept formalized for the first time by Holland [12]. It emulates the natural selection and the evolution mechanism of Darwin. The genetic algorithm proved among all of these evolutionary algorithms that it has best performance and provide more precisely and efficiency for solving the combinatorial optimization problems (COP). Within our work, we planned a Genetic Improvement Concentric Tabu Algorithm(GICTA), which integrates the local technique to classical metaheuristic GA. In hybrid algorithm (presented) the local 
approach tested to the best individual solution obtained by GA in order to protect the correspondence due to search process between both exploration and exploitation [13]. The genetic algorithm tries to be more effective for finding best population while the goal of local method is to provide better solution by testing all its neighboring and selecting the best fitness. To improve the nature of metaheuristic algorithm, local technique has been inserting into it. Overall, there is no way to pick up the optimal solution [13-15]. Consequently, in evolutionary algorithms without applying local techniques there is no promise to obtain the optimal solution [16]. To check the capability of proving the proposed algorithm, a benchmark problem taken from TSPLIB [17] are used. Remain sections of this paper is formed as follows. Section two shortly introduce the traveling salesman problem. Section three describe the basic GICTA. Section four explains/discusses the results. Final section provides the conclusion, future work and expansion.

\section{TRAVELING SALESMAN PROBLEM (TSP)}

The traveling salesman problem (TSP) is the renowned quoted non deterministic polynomial hard problem in $\mathrm{CO}$, because the difficulty and challenging to solve it rather than understanding it [18]. A lot of algorithms and a various approach has been tested in order to solving the traveling salesman problem, some of them has a good performance and obtained a very well solutions, while others have limitation to solve some of very large problems. TSP describes has been done in Graph theory describes as a weighted graph where variables are characterized as follows, $\mathrm{TSP}=\mathrm{G}=(\mathrm{V}, \mathrm{E}, \mathrm{c})$ in which $\mathrm{V}=(\mathrm{v} 1, \mathrm{v} 2, . . \mathrm{vm})$, vertex is $\mathrm{vi}(1<\mathrm{i}<\mathrm{m}), \mathrm{E}=\operatorname{eij}(\mathrm{mxm})$ (edges set) where eij $(1<\mathrm{i}, \mathrm{j}<\mathrm{n})$ is the edge which contact vi and $\mathrm{vj}$, and $\mathrm{a}: \mathrm{E}-\mathrm{-}>\mathrm{Z}$, the cost or distance usually refer to c, and the cost must not override c. TSP is classified into two categories, one of them is Symmetric while the other is Asymmetric based on the graph type and arrangement of distances. If the distance in two sides is same, it is symmetric, but if not equal it is asymmetric [19].

To calculate the tour length for symmetric type is shown below in (1).

$$
L=\left(\sum_{i=1}^{n-1} \mathrm{D} c i c i+1\right)+\mathrm{D} c i c i+1
$$

The problem is opacity for finding a lowest path. By using the Euclidean Distance The distance between cities with coordinates $(\mathrm{x}, \mathrm{y})$ is calculated as follows:

$$
\mathrm{D}_{\mathrm{xy}}=\sqrt{(x-y)^{2}}
$$

\section{THE PROPOSED ALGORITHM}

A hybrid GA used as a technique for incorporation of GA with the local techniques to solve the traveling salesman problem in efficient manner. The role of the genetic algorithm is to choose best individual solution by satisfying a reverse sequence mutation, tournament selection, and an ordered crossover as well as the initial solution has been select randomly in the very beginning of the algorithm. In contrast, the role of the local method is to escape from local optima by acting and generating some action to improve the over hand solution and trying to reach the global optimal solution or sub global optimal solution depending on the scale of the problem. In large scales the proposed algorithm picks up the very near global solution, while in small and medium scales the presented algorithm easy reach to global solution in short time. The proposed Hybrid Genetic Algorithm combines the local search method into best individual solution found by the traditional metaheuristic algorithm with a view to control and protect the poise and to picks the positively advantages for both exploration and exploitation ability due to the search process [19-20]. The goal of local search is to exchanging all the outstanding solutions nearby to the finest found solution and evaluating the distance, which distance is small or have a less fitness, in order to improve it, after that it will be select as a best solution found so far. If the favorable solution is worse than to very nearby solution, it is going to exchange it, and it will become the best solution, and the process will continue until all nearby solution been checked and global or near to global solution has been taken. Heuristic genetic algorithm is tried to enhance every population by select best individual. For the above reason the important of local methods appear to obtain the best outcomes [21]. Taking into account without local techniques no method can be found for optimum solutions, and there is no guarantee to cross the local optimal, and its very time consuming without any change, and it remain in stuck as well. Therefore, evolutionary algorithms have limited opportunity run away from local solution and pick up the optimal global solution. The following hybrid algorithm illustrates the steps of finding and selecting the random initial solution until arrive to the best-found solution over all applying the algorithm for solving travelling salesman problem. 


\subsection{Algorithm-1}

a) Applying the genetic algorithm parameters (initialization, Evaluation, selection, crossover and mutation) to obtain the best individual solution.

b) The best individual solution found by the genetic algorithm it exercised to the local search method. It is set to the center solution.

c) Set count $=0, p=0$, flag $=0$. Finest list contain the middle solution. Best found solution is set to the best list as well as the best fitness is set to the best found solution fitness.

d) If flag $=0$.the iteration will stop.

e) If flag $=1$. Evaluate the center solution nearby.

f) If the fitness of mid solution is not better than to it's very near solution. Set the best found solution and its solution to the very near one.

g) Hamming distance between the outstanding found and the very near solution to it will be calculate.

h) If the outstanding found solution still better to its nearby solution, go to step 4.

i) Otherwise, set neighbor solution to the hamming distance.

j) If best solution found go to step 2 .

k) Otherwise set count=count +1 and go to step 6 (to evaluate the reaming exchanges).

1) If criteria reached, iteration stop.

m) Otherwise, go to step 1 .

\section{COMPUTATIONAL RESULTS}

In order to assess the efficiency and the capability of the GICTA. Twenty seven (27) symmetric benchmark problem have been carry out from TSPLIB. Bays29, Berlin52, Ch150, Eil101, KroA100, KroA150, KroB100, KroB150, KroC100, KroE100, and Lin105. Moreover, to take the average every problem has been examined 10 times. Genetic algorithm parameters consists of the following: Population size $=200$, mutation probability $=0.01$, crossover probability $=1$, and the generation $=200$ to terminate. To protect and to minimize time consuming computation, the local method only tested ad applied once every fifty iteration.

In Table 1. illustrates the numerical result obtained by the proposed algorithm, and it is described as follows: column one include the name of the TSP problem, column two exhibit the favorable outcomes found by the planned algorithm while the mean is get in the third column, the column four refers to the error percentage, the fifth and sixth column indicated the standard deviation as well as time executing. As shown in table below problems with limited domain they are applies in concise time and achieve to the global solution by restricted and finite moves, however problems have a large domain they were took much time get global solutions.

Table 2. Show the execution of the traditional genetic algorithm. As seen the result has been plant by the planned algorithm are much favorable compared to the result of the heuristic genetic algorithm have. This is prove that the presented algorithm has best performance comparing to the GA.

Table 3 demonstrate the best solution obtained by local search technique. As shown below the got solutions are local optimal solution. The reason for that is, the local search method cannot escape and climb from the local optimal solution. That's why only local techniques never reach to the global optimal solution. So the proposed algorithm tried to cover this issue and combined to the heuristic algorithm in order to run away from stuck. In contrast, local techniques easily reach to the local optimal solution without consuming time.

In comparison to the other researches, we have seen not all researchers uses the same instances of TSP problems. many scientists applied various instances on their algorithms. Some of the common problem tested by many of them are Berlin52, KroA100, and Lin105. As we noticed, the proposed algorithm have better respond comparing to the others [22-25].

Table 1. Hybrid Genetic Algorithm Experimental Results

\begin{tabular}{cccccc}
\hline Problem [Optimal] & $\begin{array}{c}\text { Best } \\
\text { Sol. }\end{array}$ & $\begin{array}{c}\text { Average } \\
(\text { Mean })\end{array}$ & $\begin{array}{c}\text { Error } \\
(\%)\end{array}$ & $\begin{array}{c}\text { ST } \\
\text { DEV }\end{array}$ & $\begin{array}{c}\text { Time } \\
(\text { Sec. })\end{array}$ \\
\hline Bays29 [2020] & 2020 & 2044.8 & 0 & 87 & 131 \\
Berlin52 [7542] & 7542 & 7736 & 0 & 19 & 11 \\
Ch150 [6528] & 6879 & 7019 & 0.05 & 18.9 & 2031 \\
Eil101 [629] & 632 & 665 & 0.1 & 55.6 & 934 \\
KroA100 [21282] & 21721 & 22321 & 0.02 & 312 & 1377 \\
kroA150 [26524] & 28087 & 28900 & 0.07 & 939 & 2409 \\
kroB100 [22141] & 22177 & 22773 & 0.001 & 371 & 3127 \\
kroB150 [26130] & 27419 & 28349 & 0.05 & 965 & 2257 \\
kroC100 [20749] & 21157 & 22201 & 0.01 & 641 & 1372 \\
kroE100 [22068] & 22324 & 22697 & 0.012 & 321 & 925 \\
Lin105 [14379] & 14611 & 15001 & 0.03 & 177 & 1017 \\
\hline
\end{tabular}


Table 2. Genetic Algorithm Experimental Results

\begin{tabular}{cccccc}
\hline Problem [Optimal] & $\begin{array}{c}\text { Best } \\
\text { Sol. }\end{array}$ & $\begin{array}{c}\text { Average } \\
(\text { Mean })\end{array}$ & $\begin{array}{c}\text { Error } \\
(\%)\end{array}$ & $\begin{array}{c}\text { ST } \\
\text { DEV }\end{array}$ & $\begin{array}{c}\text { Time } \\
(\text { Sec. })\end{array}$ \\
\hline Bays29 [2020] & 2020 & 2096 & 0 & 46 & 2.5 \\
Berlin52 [7542] & 7543 & 8117 & 0.0002 & 333 & 21 \\
Ch150 [6528] & 7314 & 7657 & 0.1 & 194 & 190 \\
Eil101 [629] & 685 & 710 & 0.08 & 17 & 184 \\
KroA100 [21282] & 22563 & 23733 & 0.06 & 924 & 161 \\
kroA150 [26524] & 29233 & 30330 & 0.1 & 834 & 821 \\
kroB100 [22141] & 23670 & 24245 & 0.07 & 512 & 186 \\
kroB150 [26130] & 29771 & 30983 & 0.1 & 701 & 741 \\
kroC100 [20749] & 22709 & 23669 & 0.9 & 682 & 183 \\
kroE100 [22068] & 23534 & 24443 & 0.07 & 739 & 191 \\
Lin105 [14379] & 14631 & 15675 & 0.01 & 511 & 222 \\
\hline
\end{tabular}

Table 3. Local Search Algorithm Experimental Results

\begin{tabular}{cccccc}
\hline $\begin{array}{c}\text { Problem } \\
\text { Opptimal] }\end{array}$ & $\begin{array}{c}\text { Best } \\
\text { Sol. }\end{array}$ & $\begin{array}{c}\text { Average } \\
(\text { Mean })\end{array}$ & $\begin{array}{c}\text { Error } \\
(\%)\end{array}$ & $\begin{array}{c}\text { ST } \\
\text { DEV }\end{array}$ & $\begin{array}{c}\text { Time } \\
(\text { Sec. })\end{array}$ \\
\hline Bays29 [2020] & 2153 & 2305 & 0.06 & 86 & 2.7 \\
Berlin52 [7542] & 8035 & 8862 & 0.06 & 445 & 5.7 \\
Ch150 [6528] & 9398 & 9803 & 0.4 & 215 & 27 \\
Eil101 [629] & 715 & 752 & 0.1 & 215 & 14 \\
KroA100 [21282] & 27585 & 30193 & 0.3 & 1306 & 15 \\
kroA150 [26524] & 38273 & 41303 & 0.45 & 1565 & 29 \\
kroB100 [22141] & 28107 & 31003 & 0.3 & 1315 & 25 \\
kroB150 [26130] & 37855 & 41375 & 0.46 & 2525 & 29 \\
kroC100 [20749] & 27250 & 29729 & 0.3 & 1605 & 14 \\
kroE100 [22068] & 28991 & 30065 & 0.3 & 745 & 24 \\
Lin105[14379] & 20170 & 21840 & 0.4 & 1190 & 14 \\
\hline
\end{tabular}

\section{CONCLUSION}

To conclude this paper, a hybrid genetic algorithm (integration of both local technique and heuristic GA) tested to the symmetric Euclidean traveling salesman problem. By comparing to the traditional genetic algorithm experimental shows the proposed algorithm has a better response as well as its improved the performance by making them to desertion from the regional or local favorable and search for the most favorable or near to it, and shows that it's has excellent execution for a certain scope.

\section{REFERENCES}

[1] E. Osaba, R. Carballedo, F. Diaz, and A. Perallos, "Analysis of the suitability of using blind crossover operators in genetic algorithms for solving rouning problems". IEEE International Symposium on Applied Computational Intelligence and Informatics, 2013, pp. 17-22.

[2] P. Chang, W. Huang, C. Ting, and W. Chang, "A varietal genetic algorithm by external self-evolving multiple-archives for combinatorial optimization problems". Proceedings of the 11th IEEE International Conference on High Performance Computing and Communications, 2009, pp. 609-614.

[3] Al-Obaidi, A. T. S., Abdullah, H. S., \& Ahmed, Z. O. Meerkat clan algorithm: A new swarm intelligence algorithm. Indonesian Journal of Electrical Engineering and Computer Science, 2018, pp. 354-360.

[4] S. Shubhra, B. Sanghamitra, and K. Sankar. "New Operators of Genetic Algorithms for Traveling Salesman Problem." International Conference on Pattern Recognition, 2004, pp. 497-500.

[5] J. Grefenstette, R. Gopal, B. Rosimaita, and D. Gucht, "Genetic Algorithms for the Traveling Salesman Problem." Proc. Int. Conf. Genetic Algorithms and Their Applications, July 1985, pp. 160-168.

[6] S. Kirkpatrick, Jr. Gelatt, and M. Vecchi, “Optimization by Simulated Annealing. Science,” 1983, 498-516.

[7] Inggih P., Nesdi E., Fadhilah S., \& Febi Nur Salisah. "Optimization Learning Vector Quantization Using Genetic Algorithm for Detection of Diabetics.” Indonesian Journal of Electrical Engineering and Computer Science, 2018, pp. 1111-1116.

[8] J. Kennedy and R. Eberhart, "Particle swarm optimization". Proceedings of IEEE International Conference on Neural Networks, 1995, pp. 1942-1948.

[9] F. Glover, “Tabu Search,” Part I. ORSA Journal on Computing, 1989, pp. 190-206.

[10] Eesa, A. S., Orman, Z., \& Brifcani, A. M. A. "A new feature selection model based on ID3 and bees algorithm for intrusion detection system." Turkish Journal of Electrical Engineering \& Computer Sciences, 2015, pp. 615-622.

[11] S. Jayalakshmi, R. Aswini. "A Novel Optimization Algorithm Based on Stinging Behavior of Bee." IAES International Journal of Artificial Intelligence (IJ-AI), 2018, pp. 153-164. 
[12] D. Pham, and T. Huynh, "an Effective Combination of Genetic Algorithms and the Variable Neighborhood Search for Solving Travelling Salesman Problem". IEEE Conference on Technologies and Applications of Artificial Intelligence, 2015, pp. 142-149.

[13] D. Zvi, "Tabu Search and Hybrid Genetic Algorithms for Quadratic Assignment Problems." European Journal of Operational Research, 2008, pp. 90-107.

[14] D. Zeebaree, H. Haron, A. Muhsin, and S. Zeebaree. "Combination of K-means clustering with Genetic Algorithm: A review." International Journal of Applied Engineering Research, 2017, pp. 14238-14245.

[15] Xingwang H., Xuewen Z., Rui H., \& Xu W. "An enhanced hybridized artificial bee colony algorithm for optimization problems." IAES International Journal of Artificial Intelligence (IJ-AI), 2019, pp. 87-94.

[16] J. Potvin, "The Traveling Salesman Problem: A Neural Network Perspective." ORSA J. Comput. 1993, pp. 328-348.

[17] http://elib.zib.de/pub/mp-testdata/tsp/tsplib/tsp/index.html.

[18] Arananayakgi, "Reduce Total Distance and Time Using Genetic Algorithm." International Journal of Computer Science and Engineering Technology, 2014, pp. 815-819.

[19] J. Holland, "Adaptation in Natural and Artificial Systems: An Introductory Analysis with Applications to Biology, Control, and Artificial Intelligence.” University Michigan Press, Ann Arbor, MI, USA, 1975.

[20] Eesa, A. S., Orman, Z., \& Brifcani, A. M. A. "A novel feature-selection approach based on the cuttlefish optimization algorithm for intrusion detection systems.” Expert Systems with Applications, 2015, pp. 2670-2679.

[21] Surakhi, O., Khanafseh, M., \& Jaffal, Y. "An enhanced Biometric-based Face Recognition System using Genetic and CRO Algorithms."

[22] Y. Deng, Y. Liu, and D. Zhou. “An Improved Genetic Algorithm with Initial Population Strategy for Symmetric TSP.” Mathematical Problems in Engineering, 2015, pp. 791-797.

[23] P. Chann, W. Hsiu, \& C. Jung. "Dynamic diversity control in genetic algorithm for mining unsearched solution space in TSP problems.” Expert Systems with Applications, 2010, pp. 1863-1878.

[24] F.Hanif, N. Khan, S. Inayatullah, \& S. Tajuddin. "Solving TSP Problem by Using Genetic Algorithm." International Journal of Basic \& Applied Sciences, 2009.

[25] Y. Yu, Y. Chen and T. Li. "A New Design of Genetic Algorithm for Solving TSP.” 2011 Fourth International Joint Conference on Computational Sciences and Optimization, 2011, pp. 309-313. 